\title{
Entanglement Scaling in the One-Dimensional Hubbard Model at Criticality
}

\author{
Daniel Larsson and Henrik Johannesson \\ Department of Physics, Göteborg University, SE 41296 Göteborg, Sweden
}

\begin{abstract}
We derive exact expressions for the local entanglement entropy $\mathcal{E}$ in the ground state of the onedimensional Hubbard model at a quantum phase transition driven by a change in magnetic field $h$ or chemical potential $\mu$. The leading divergences of $\partial \mathcal{E} / \partial h$ and $\partial \mathcal{E} / \partial \mu$ are shown to be directly related to those of the zero-temperature spin and charge susceptibilities. Logarithmic corrections to scaling signal a change in the number of local states accessible to the system as it undergoes the transition.

PACS numbers: 71.10Fd,03.65.Ud,03.67.Mn,05.70.Jk
\end{abstract}

Entanglement is a generic feature of quantum systems, implying the possible existence of non-local correlations. Such correlations - which lead to highly counter-intuitive phenomena - were long seen as an artifact of quantum mechanics [1]. With the advent of quantum information theory it is now understood that entanglement - and the correlations associated with it - is not only intrinsic to the fabric of reality [2], but can also be used as a physical resource, essential for performing such tasks as teleportation or quantum computing 3 ].

A new line of research [4, 5] points to a connection between the entanglement of a many-particle system as quantified by a properly chosen measure - and the appearance of a (zero-temperature) quantum phase transition (QPT) 6]. Barring accidental occurrences of nonanalyticity, a discontinuity [singularity] in the [derivative of the] ground state concurrence of an $N$-qubit system appears to be associated with a first [second] order QPT []] (with concurrence measuring the entanglement between two neighboring qubits [8]). These and related results are important as they hold promise of novel perspectives on condensed matter, drawing on insights from quantum information theory. By analyzing entanglement properties one expects to gain insight into how the associated non-local (purely quantum) correlations influence the critical behavior of a quantum phase transition. Building an understanding of this connection should enable breakthroughs in the design of future experimental probes of collective quantum phenomena. Also, architectures for quantum information processing that take advantage of the entanglement in the vicinity of a quantum phase transition (quantum adiabatic computing) [9] should benefit from a detailed understanding of entanglement scaling properties.

Most results to date on the entanglement - QPT connection have been obtained from numerical studies of finite lattice spin systems, supplemented by some analytical results [10]. Much less is known about entanglement scaling properties of itinerant electron systems. In this Letter we make a dent on this important problem by studying the one-dimensional Hubbard model close to a quantum phase transition. Recent work on this and related models show that features of the ground state phase diagram can be reproduced by studying certain characteristics of the local entanglement entropy 11, 12, 13, 14, 15. Here we exploit the Bethe Ansatz solvability of the Hubbard model to derive exact expressions for the critical scaling of the local entanglement entropy $\mathcal{E}\left(\psi_{0}\right)$ of its ground state $\left|\psi_{0}\right\rangle$ as function of magnetic field $h$ and chemical potential $\mu$. We find that the leading scaling behavior of $\partial \mathcal{E} / \partial \mu$ for repulsive interaction coincides with that of the charge susceptibility $\chi_{C}$. A similar result holds for $\partial \mathcal{E} / \partial h$, but with logarithmic corrections that signal a change of dimension of the accessible local state space at the transition. The fact that an entanglement measure of a critical many-particle system can be quantitatively linked to a physical observable is a striking result, and goes beyond standard constructions of entanglement witnesses 16] that merely detect the presence of entanglement. To what extent our results can be generalized to other quantum systems is yet to be answered.

To set the stage, let us recall that the very notion of entanglement of a composite quantum system relies on the tensor product structure of its Hilbert space. When the system is made up of itinerant electrons, however, the physical subspace is restricted to an anti-symmetrized one which lacks a natural product structure. One may circumvent the problem by passing to an occupation number representation spanned by the $4^{L}$ basis states $|n\rangle_{1} \otimes|n\rangle_{2} \otimes \ldots \otimes|n\rangle_{L}$, where, in obvious notation, $|n\rangle_{j}=|0\rangle_{j},|\uparrow\rangle_{j},|\downarrow\rangle_{j}$, or $|\uparrow \downarrow\rangle_{j}$ is a local state at site $j$, with $L$ the number of sites on the lattice [17]. This is a convenient basis in which the tensor product structure is manifestly recovered, with the local states describing electronic modes easily accessible to an observer. By splitting the system into two parts $A$ and $B$ one can then proceed as usual and define the entanglement entropy $\mathcal{E}$ of a pure state $|\psi\rangle$ as $\mathcal{E}=-\operatorname{Tr}\left(\rho_{A} \log _{2} \rho_{A}\right)$ ] 3 . The reduced density matrix $\rho_{A}=\operatorname{Tr}_{B}(\rho)$ is obtained from the full density matrix $\rho=|\psi\rangle\langle\psi|$ by tracing out the local states belonging to $B$. In what follows we focus on the entanglement entropy of a single site, obtained by taking $A$ to be a single (arbitrarily chosen) site, with $B$ the rest of the system.

We begin by studying the Hubbard model with an ap- 
plied magnetic field $H$ :

$$
\mathcal{H}=-t \sum_{\substack{j=1 \\ \delta= \pm 1}}^{L} c_{j \alpha}^{\dagger} c_{j+\delta \alpha}+U \sum_{j=1}^{L} n_{j \uparrow} n_{j \downarrow}-\mu_{B} H \sum_{j=1}^{L} S_{j}^{z} .
$$

Here $c_{j \alpha}^{\dagger}$ and $c_{j \alpha}$ are creation and annihilation operators for electrons at site $j$ with spin $\alpha=\uparrow, \downarrow$, and $n_{j \alpha} \equiv c_{j \alpha}^{\dagger} c_{j \alpha}$ and $S_{j}^{z}=\left(n_{j \uparrow}-n_{j \downarrow}\right) / 2$ are the corresponding number and spin operators, respectively. We assume periodic boundary conditions. In the following we shall work with dimensionless quantities $u \equiv U / 4 t$ and $h \equiv \mu_{B} H / t$, putting $t=1$ (of dimension energy). Using the fact that the Hamiltonian in (11) is translational invariant and conserves particle number as well as the z-component of the total spin, it is easy to verify that the reduced density matrix $\rho_{A}$ for a single site $A$ is diagonal in the chosen basis. It follows that the corresponding single-site entanglement of the ground state $\left|\psi_{0}\right\rangle$ can be written as

$$
\mathcal{E}=-w_{0} \log _{2} w_{0}-w_{\uparrow} \log _{2} w_{\uparrow}-w_{\downarrow} \log _{2} w_{\downarrow}-w_{2} \log _{2} w_{2}
$$

with

$$
\begin{aligned}
& w_{2}=\left\langle n_{j \uparrow} n_{j \downarrow}\right\rangle_{0}, \quad w_{\alpha}=\left\langle n_{j \alpha}\right\rangle_{0}-w_{2}, \alpha=\uparrow, \downarrow \\
& w_{0}=1-w_{\uparrow}-w_{\downarrow}-w_{2} .
\end{aligned}
$$

The problem is thus reduced to calculating the expectation values for double and single (spin-up and spin-down) occupancies in the ground state.

Let us first look at the case of attractive interaction, $u<0$, with $n=1$ (half-filling). In the limit $|u| \gg 1$ we can use the Hellman-Feynman theorem, $\langle\partial H / \partial u\rangle_{0}=$ $\partial E_{0} / \partial u$, together with the known Bethe Ansatz result for the ground state energy [18], $E_{0} / 4 L=u(1 / 2-m)-$ $(1 / 2 \pi) \sin (2 \pi m)+\mathcal{O}(1 / u)$ to obtain

$$
w_{2}=\frac{1}{4 L} \frac{\partial E_{0}}{\partial u}=\frac{1}{2}-m+\mathcal{O}\left(1 / u^{2}\right),
$$

with $m=(1 / 2 L) \sum_{j=1}^{L}\left\langle n_{j \uparrow}-n_{j \downarrow}\right\rangle$ the magnetization per site. Neglecting the $\mathcal{O}\left(1 / u^{2}\right)$ corrections it follows immediately from (33) and (4) that

$$
w_{\uparrow}=2 m, \quad w_{\downarrow}=0, \quad w_{0}=\frac{1}{2}-m, \quad h \geq 0 .
$$

Combining (2), (4), and (5), we obtain for the single-site entanglement:

$$
\mathcal{E}=-2 m \log _{2}(2 m)-(1-2 m) \log _{2}\left(\frac{1}{2}-m\right), \quad h \geq 0 .
$$

The dependence of the magnetization on the applied field can also be derived from the ground state energy, and one finds

$$
m(h)= \begin{cases}0 & 0 \leq h<h_{c 1} \\ \frac{1}{2 \pi} \arccos \left(-\left(u+\frac{h}{4}\right)\right) & h_{c 1} \leq h \leq h_{c 2} \\ \frac{1}{2} & h_{c 2}<h\end{cases}
$$

with lower [upper] critical field $h_{c 1}=4(|u|-1)\left[h_{c 2}=\right.$ $4(|u|+1)]$. The single-site entanglement as a function of magnetic field, $\mathcal{E}=\mathcal{E}(h)$, can now be read off from (6) and (7). The result for the $|u| \gg 1$ limit is plotted in Fig. (11) for large values of $h$. Note that in this limit there are two local states, $|0\rangle$ and $|\uparrow \downarrow\rangle$, available to the system when $h<h_{c 1}$, implying that $\mathcal{E}(h)=1$. In contrast, the fully magnetized state for $h>h_{c 2}$ is a direct product of local spin-up states, and hence $\mathcal{E}(h)=0$. For comparison we have plotted the single-site entanglement for free electrons also in Fig. (11) (for both positive and negative values of the magnetic field). This result is easily obtained from Ref. [18] by noting that $w_{2}=1 / 4-m^{2}$ when $u=0$, with $m=(1 / \pi) \arcsin (h / 4)$ in the interval $-4<h<4$.

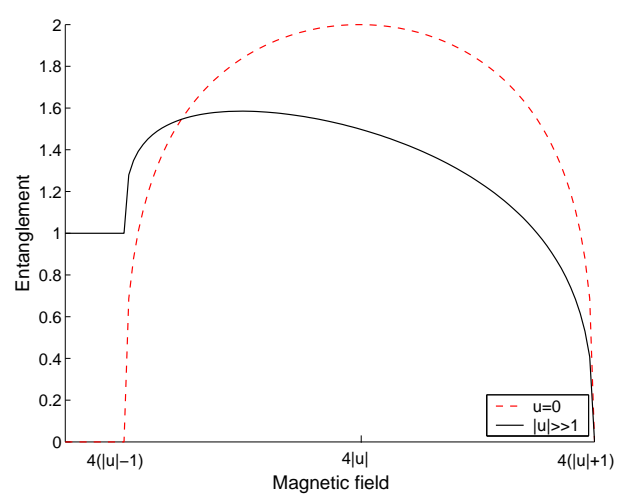

FIG. 1: Entanglement entropy $\mathcal{E}$ of a single site versus magnetic field $h$ for the attractive Hubbard model with $|u| \gg 1$ (solid curve). For comparison, the single-site entanglement for the free case $(u=0)$ is shown by the dotted curve (on a different scale).

The phase transitions at $h_{c 1}$ and $h_{c 2}$ are second order, with diverging spin susceptibilities $\chi_{S i}=\left(32 \pi^{2} \mid h-\right.$ $\left.h_{c i} \mid\right)^{-1 / 2}, i=1,2[18$ ]. As mentioned in the introduction, in the case of an $N$-qubit system there is strong evidence that the derivative of the ground state concurrence with respect to a critical parameter diverges at a second-order QPT [7]. For the present problem (where the local degrees of freedom have four components, not two as for a qubit) the plot in Fig. (11) suggests a divergence of $\partial \mathcal{E} / \partial h$ as $h \rightarrow h_{c 1+}$ and $h \rightarrow h_{c 2-}$. To analytically check whether the derivative of the single-site entanglement is a true marker of quantum criticality for this problem, we write $u+h / 4=\left(h-h_{c i}\right) / 4+(-1)^{i}, i=1,2$ and expand $\partial \mathcal{E} / \partial h$ in $h-h_{c 1}$ and $h_{c 2}-h$, respectively. We obtain

$$
\frac{\partial \mathcal{E}}{\partial h}=(-1)^{i} \frac{\chi_{S i}}{\ln (2)}\left(\ln \left|h-h_{c i}\right|+\text { const. }\right), \quad i=1,2
$$

for $h \rightarrow h_{c 1+}$ and $h \rightarrow h_{c 2-}$, respectively. This confirms that $\partial \mathcal{E} / \partial h$ diverges at the magnetic phase transitions. Moreover, it shows that the divergence of $\partial \mathcal{E} / \partial h$ is given by the spin susceptibility - up to a logarithmic correction. 
By writing the leading term of $\partial \mathcal{E} / \partial h$ on the "mixed" form $(-\partial m / \partial h)\left(2 \ln \left(w_{\uparrow}\right)-\ln \left(w_{0}\right)-\ln \left(w_{2}\right)\right) / \ln 2$ [cf. Eqs. (21) and (6)], and combining this expression with (7), one sees that the logarithmic divergence in (8) comes from a change of the number of local states accessible to the system as it undergoes the transition: As $h \rightarrow h_{c 1+}$ the local spin-up states get suppressed $\left(w_{\uparrow} \rightarrow 0\right)$, while for $h \rightarrow h_{c 2-}$ both empty and doubly occupied local states get suppressed $\left(w_{0}, w_{2} \rightarrow 0\right)$.

Turning to the half-filled case with repulsive interaction, $u>0$, a QPT now occurs only at the value of the field for which the magnetization saturates: $h_{c 2}=$ $4\left(\sqrt{1+u^{2}}-u\right)[19]$. As shown by Takahashi, the ground state energy for any finite value of $u>0$ in the critical region $h \rightarrow h_{c 2-}$ can be expanded in terms of the expectation value for single spin-down occupancy [20]:

$$
\begin{aligned}
\frac{E_{0}}{4 L} & =-\left(\sqrt{1+u^{2}}-u\right)\left\langle n_{j \downarrow}\right\rangle_{0} \\
& +\frac{\pi^{2}}{24} \frac{1}{\sqrt{1+u^{2}}}\left\langle n_{j \downarrow}\right\rangle_{0}^{3}+\mathcal{O}\left(\left\langle n_{j \downarrow}\right\rangle_{0}^{4}\right) .
\end{aligned}
$$

With the same procedure as used for the attractive case above, Eq. (9), together with (2) and (3), yield:

$$
\frac{\partial \mathcal{E}}{\partial h}=\frac{C}{2 \ln (2)} \chi_{S}\left(\ln \left|h-h_{c 2}\right|+\text { const. }\right), \quad h \rightarrow h_{c 2-} .
$$

Here $C=2-u / \sqrt{1+u^{2}}$, and $2 \pi \chi_{S}=\left(4+4 u^{2}\right)^{1 / 4} \mid h-$ $\left.h_{c 2}\right|^{-1 / 2}$. The logarithmic correction in (10) now signals the suppression of all but the spin-up states as one approaches the saturation point $h_{c 2}$ from below.

We next study the effect of a varying chemical potential on the single-site entanglement of an open system. We make the simplifying assumption that the environment acts solely as a particle reservoir 21], and add the term $\mathcal{H}_{\mu}=-\mu \sum_{j=1}^{L}\left(n_{j \uparrow}+n_{j \downarrow}\right)$ to the Hamiltonian in (1), with $\mu$ a dimensionless chemical potential (multiplied by the hopping amplitude $t=1$ ). To simplify further we turn off the magnetic field in (1), putting $h=0$. Focusing on the case of repulsive interaction, $u>0$, with $n \leq 1$, the system exhibits two quantum critical points [22]: $\mu_{c 1}=-2$ and $\mu_{c 2}=2-4 \int_{0}^{\infty} J_{1}(\omega)(\omega[1+\exp (2 \omega u)])^{-1} d \omega$, with $J_{1}(\omega)$ a first-order Bessel function. Both transitions are second-order with diverging charge susceptibilities $\chi_{C i}=c(u)\left|\mu-\mu_{c i}\right|^{-1 / 2}, i=1,2$ in the limits $\mu \rightarrow \mu_{c 1+}$ (empty lattice transition) and $\mu \rightarrow \mu_{c 2-}$ (Mott transition), respectively (with $c(u)$ a positive $u$-dependent constant). To obtain the single-site entanglement $\mathcal{E}$ we first notice that $\mathcal{H}_{\mu}$ conserves spin and particle number for fixed $\mu$, and that hence the expression for $\mathcal{E}$ in (2) remains valid. Recalling from the Lieb-Mattis theorem 23. that the ground state has zero spin (for any $n$ with $n L$ an even integer) we can write the parameters appearing in (2) as

$$
w_{0}=1-n+w_{2}, \quad w_{\uparrow}=w_{\downarrow}=\frac{n}{2}-w_{2} .
$$

The value of $w_{2}$ can again be extracted from the ground state energy via the relation $w_{2}=\left(\partial E_{0} / \partial u\right) / 4 L$, where the Bethe Ansatz solution for $E_{0}$ can now be expressed via a $1 / u$ expansion [24]:

$$
\frac{E_{0}}{L}=-\frac{2}{\pi} \sin (\pi n)-\sum_{l=1}^{\infty} \kappa_{l}(n)\left(\frac{1}{4 u}\right)^{l} .
$$

The values of $\kappa_{l}(n)$ are tabulated to fifth order in Ref. [24]. The ground state energy in (12) also determines the chemical potential as function of filling: $\mu(n)=\partial E_{0} / \partial n$. By inverting $\mu(n)$ and inserting the resulting values for the $w$-parameters from (3) into (2) we can plot $\mathcal{E}$ vs. $\mu$ for any value of $u>1$. Some representative plots are shown in Fig. (2), together with the single-site entanglement for free electrons $(u=0)$.

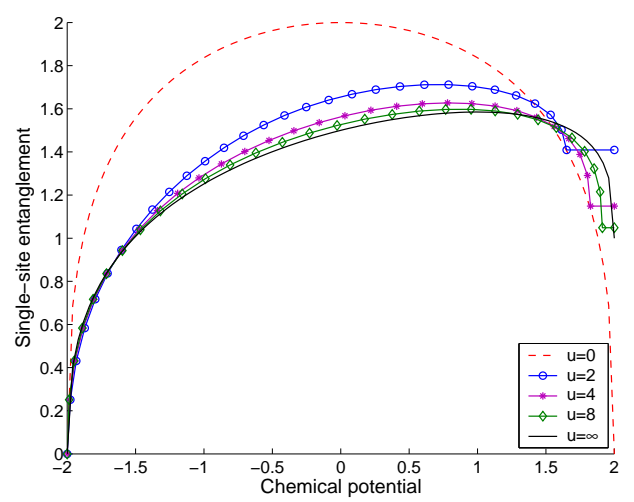

FIG. 2: Entanglement entropy $\mathcal{E}$ of a single site versus chemical potential $\mu$ for the repulsive Hubbard model. The plateaus correspond to half-filling $(n=1)$, cut off at $\mu=2$. The dotted curve is that for free electrons $(u=0)$, plotted in the region $0 \leq n \leq 2$.

In order to analytically explore the quantum critical regions $\mu \rightarrow \mu_{c 1+}$ and $\mu \rightarrow \mu_{c 2-}$ we first consider the $u \rightarrow \infty$ limit where $w_{2}=0$. In this limit (12) implies that $n(\mu)=(1 / \pi) \arccos (-\mu / 2)$. Combining this expression with Eqs. (2) and (11) we obtain

$$
\frac{\partial \mathcal{E}}{\partial \mu}=(-1)^{i} \frac{\chi_{C i}}{2 \ln (2)}\left(\ln \left|\mu-\mu_{c i}\right|+\text { const. }\right), i=1,2
$$

for $\mu \rightarrow \mu_{c 1+}$ and $\mu \rightarrow \mu_{c 2-}$, respectively. Note that the derivative of the single-site entanglement is again given by a susceptibility, corrected by a logarithmic factor that reflects the change in the number of available local states as the system undergoes the transition: When $\mu \rightarrow \mu_{c 1+}\left[\mu \rightarrow \mu_{c 2-}\right]$ the singly occupied [empty] local states get suppressed. (Cf. the argument after Eq. (8).) The exact analogy with the magnetic scaling in (8) can be understood by carrying out a particle-hole transformation for spin-up electrons: $c_{j \uparrow}^{\dagger} \leftrightarrow(-1)^{j} c_{j \uparrow}$ (leaving the spin-down electrons untouched). This transformation maps the zero-field attractive system with a variable 
chemical potential onto a half-filled repulsive system with a variable magnetic field. It follows that the single-site entanglement at $\mu_{c 1}\left(\mu_{c 2}\right)$ has the same behavior as at $h_{c 2}\left(h_{c 1}\right)$.

Turning to the case of large but finite $u$, we focus on the Mott transition $\mu \rightarrow \mu_{c 2-}$. A straightforward analysis, again using the Bethe Ansatz result in (12), yields for the leading behavior of the single-site entanglement:

$$
\frac{\partial \mathcal{E}}{\partial \mu}=-C(u) \chi_{C 2},
$$

with $C(u)$ a positive $u$-dependent constant. Note that there is no logarithmic correction in (14): When $u$ is finite the metallic $\left(\mu<\mu_{c 2}\right)$ and insulating $\left(\mu>\mu_{c 2}\right)$ ground states for $0 \leq n \leq 1$ are both superpositions of all four types of local states $|0\rangle_{j},|\uparrow\rangle_{j},|\downarrow\rangle_{j}$, and $|\uparrow \downarrow\rangle_{j}$. It follows that none of the $w$-parameters in (2) tend to zero, and the logarithmic terms add up to a constant [25].

The results in (13) and (14) derived for $0 \leq n \leq 1$ can be extended to the region $1 \leq n \leq 2$ via the particlehole transformation $c_{j \alpha}^{\dagger} \leftrightarrow(-1)^{j} c_{j \alpha}, \alpha=\uparrow, \downarrow$. One finds that the change of $\mathcal{E}$ at the quantum critical point $\mu_{c 3}=$ $4 u-\mu_{c 2}\left[\mu_{c 4}=4 u-\mu_{c 1}\right]$ where the system goes to more than half-filling, $n>1$ [complete filling, $n=2$ ] exhibits the same scaling as at the transition to half-filling [empty lattice] studied above.

To summarize, we have found that the derivatives $\partial \mathcal{E} / \partial h$ and $\partial \mathcal{E} / \partial \mu$ of the single-site entanglement $\mathcal{E}$ are faithful markers for QPTs in the Hubbard model driven by a change in magnetic field $h$ or chemical potential $\mu$. Via an analysis based on the Bethe Ansatz solution of the model, we have derived exact expressions for $\partial \mathcal{E} / \partial h$ and $\partial \mathcal{E} / \partial \mu$ at criticality, revealing that these quantities scale with the corresponding diverging spin and charge susceptibilities, respectively. Logarithmic corrections signal a change in the number of available local states at the QPT. That a critical entanglement entropy is directly connected to a susceptibility is an intriguing property. A zero-temperature susceptibility is an observable determined only by the dependence of the ground state energy on the critical parameter (magnetic field or chemical potential). The entanglement entropy, on the other hand, carries information about the very structure of the ground state. From our analysis it is transparent how the linkage formally comes about for the Hubbard model: The reduced density matrix can be parameterized by expectation values derivable from the ground state energy via the Hellman-Feynman theorem. To find a physical interpretation of the connection, and to explore whether it can be extended to other critical quantum many-particle systems, is an interesting and challenging problem.

We thank R. Rehammar and O. Syljuåsen for valuable discussions. We are also grateful to K. Capelle and V. V. França for drawing our attention to a numerical error in the original Fig. 2 of this paper (published in Phys. Rev. Lett. 95, 196406 (2005)) 26]. H.J. acknowledges support from the Swedish Research Council.

[1] A. Einstein, B. Podolsky, and N. Rosen, Phys. Rev. 47, 777 (1935).

[2] J. S. Bell, Physics 1, 195 (1964).

[3] M. A. Nielsen and I. L. Chiang, Quantum Computation and Quantum Information (Cambridge University Press, Cambridge, 2000).

[4] A. Osterloh, L. Amico, G. Falci, and R. Fazio, Nature 416, 608 (2002).

[5] T. J. Osborne and M. A. Nielsen, Phys. Rev. A 66, 032110 (2002).

[6] S. Sachdev, Quantum Phase Transitions (Cambridge University Press, Cambridge, 1999).

[7] L.-A. Wu, M. S. Sarandy, and D. A. Lidar, Phys. Rev. Lett. 93, 250404 (2004).

[8] W. K. Wootters, Phys. Rev. Lett. 80, 2245 (1998).

[9] J. I. Latorre and R. Orús, Phys. Rev. A 69, 062302 (2004).

[10] See J. I. Latorre, E. Rico and G. Vidal, Quant. Inf. and Comp. 4, 48 (2004), and references therein.

[11] V. E. Korepin, Phys. Rev. Lett. 92, 096402 (2004).

[12] S.-J. Gu, S.-S. Deng, Y.-Q. Li, and H.-Q. Lin, Phys. Rev. Lett. 93, 086402 (2004)

[13] A. Anfossi, P. Giorda, A. Montorsi, and F. Traversa, Phys. Rev. Lett. 95, 056402 (2005).

[14] A. Anfossi, C. D. E. Boschi, A. Montorsi, and F. Ortolani, Phys. Rev. B 73, 085113 (2006).

[15] For an effort to study entanglement scaling in the twodimensional Hubbard model, see J. Wang and S. Kais, Phys. Rev. A 70, 022301 (2004); quant-ph/0405085

[16] B. M. Terhal, Phys. Lett. A 271, 319 (2000).

[17] P. Zanardi, Phys. Rev. A 65, 042101 (2002).

[18] C. Yang, A. N. Kocharian, and Y. L. Chiang, J. Phys. Cond. Mat. 12, 7433 (2000).

[19] M. Takahashi, Thermodynamics of One-Dimensional Solvable Models (Cambridge University Press, Cambridge, 1999).

[20] M. Takahashi, Prog. Theor. Phys. 42, 1098 (1969).

[21] Note that a pure state measure of entanglement is still applicable at zero temperature when there is no dynamic interaction with the environment.

[22] E. H. Lieb and F. Y. Wu, Phys. Rev. Lett. 20, 1445 (1968); Physica A 321, 1 (2003).

[23] E. Lieb and D. Mattis, Phys. Rev. 125, 164 (1962).

[24] J. Carmelo and D. Baeriswyl, Phys. Rev. B 37, 7541 (1988).

[25] In Ref. 12], the change of the local entanglement of the 1D Hubbard model was found to exhibit a finite discontinuity at the Mott transition at $n=1$, and it was argued that this is a characteristic of the transition. It is important to note, however, that the discontinuity at $n=1$ is a mere consequence of the fact that the limits $n \rightarrow 1^{-}$ and $n \rightarrow 1^{+}$correspond to distinct regions for the chemical potential, $\mu \rightarrow \mu_{c 2-}$ and $\mu \rightarrow \mu_{c 3+}$, respectively. In fact, what matters is not the change of the entanglement with $n$, but with $\mu$ (which is the experimentally controllable parameter). As shown by our exact results, $\partial \mathcal{E} / \partial \mu$ 
exhibits divergences at $\mu_{c 2}$ and $\mu_{c 3}$, suggesting that the proposed classification of second order QPTs by Wu et al. 7] may be extended to local entanglement measures for fermionic systems.
[26] The corrected Fig. 2 will be published in an Erratum to Phys. Rev. Lett. 95, 196406 (2005). 